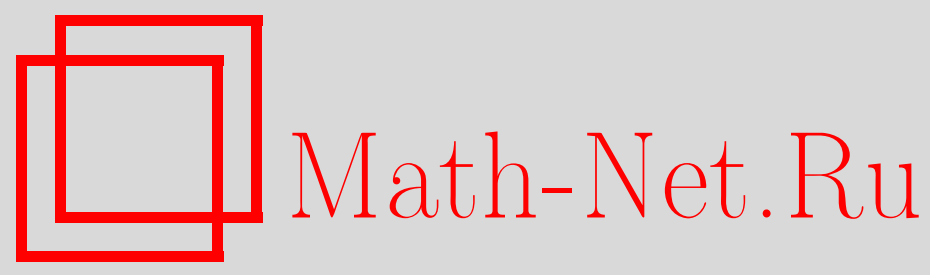

Н. Б. Ускова, К одному результату Р. Тернера, Матем. заметки, 2004, том 76, выпуск 6, 905-917

DOI: https://doi.org/10.4213/mzm162

Использование Общероссийского математического портала Math-Net.Ru подразумевает, что вы прочитали и согласны с пользовательским соглашением http://www.mathnet.ru/rus/agreement

Параметры загрузки:

IP : 34.229 .45 .116

26 апреля 2023 г., 14:05:06

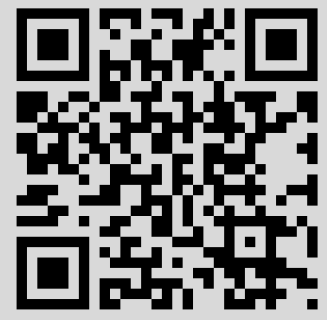




\section{К ОДНОМУ РЕЗУЛЬТАТУ Р. ТЕРНЕРА}

\section{Н. Б. Ускова}

Рассматривается вопрос о подобии возмущенного компактного оператора оператору блочно-диагональной структуры относительно некоторого семейства ортопроекторов, что позволяет уточнить и существенным образом усилить результаты Р. Тернера. Получена информация об операторе, осуществляющем преобразование подобия, приведены оценки на собственные значения и собственные векторы возмущенного оператора, а также рассмотрена обратная задача спектрального анализа.

Библиограффия: 7 названий.

В монографии [1, гл. 20, 3 3] приведены результаты, принадлежащие Р. Тернеру. Они касаются диагонализации некоторых классов компактных операторов и содержатся в следующей теореме.

Теорема 1. Пусть $H$ - сепарабельное гильбертово пространство и $A$ - нормальный компактный оператор в $H$. Пусть $\left\{e_{n}\right\}-$ ортонормированный базис в H $и A e_{n}=\lambda_{n} e_{n}$. Предположим, что в последовательности $\lambda_{n}$ нет повторений, все собственные значения $\lambda_{n}$ отличны от нуля $u r_{n}$ - расстояние от $\lambda_{n}$ до остальных точек спектра оператора $A$. Обозначим через $R$ замкнутый линейный оператор с плотной областью определения $D(R)$, которая состоит из всех векторов $x=\sum_{n=1}^{\infty} x_{n} e_{n}$ из $H$, удовлетворяюших условию

$$
\sum_{n=1}^{\infty} r_{n}^{-2} x_{n}^{2}<\infty,
$$

оператор $R$ действует по формуле

$$
R x=\sum_{n=1}^{\infty} r_{n}^{-1} x_{n} e_{n} .
$$

Пусть $M$ - мнохество всех таких ограниченных операторов, что $B x \in D(R)$ для всех $x \in H$ и RB принадлежит классу Гильберта-Шмидта $\sigma_{2}(H)$. Тогда существует такая полохительная постоянная $\varepsilon(A)$, зависящая только от оператора $A$, что если $B \in M u\|R B\|_{2}<\varepsilon(A)$, то оператор $A+B$ подобен некоторому оператору $A_{1}$, коммутирующему $с A$.

Работа выполнена при финансовой поддержке Российского фонда фундаментальных исследований, грант № 01-01-00408. 
СлЕДСТВИЕ 1. Если выполнены условия теоремы 1, то $A+B$ - спектральный оператор скалярного типа.

Данная статья посвящена усилению результата Тернера. В частности, снято условие простоты всех собственных значений, вьписан явный вид константы $\varepsilon(A)$ и оператора $A_{1}$, получена дополнительная информация об операторе, осуществляющем преобразование подобия. Более того, рассмотрен не только случай, когда $R B \in \sigma_{2}(H)$, но и другие виды возмущений. Приведены также оценки на собственные значения и собственные векторы возмущенного оператора и затронута обратная задача спектрального анализа.

Методом исследования является метод подобных операторов, состоящий в преобразовании подобия возмущенного оператора оператору более простой структуры, спектральные свойства которого легко изучать, так как они обычно бывают близки к спектральньм свойствам невозмущенного оператора. Приведенная выше теорема 1 - это один из первых результатов, которьй был получен методом подобных операторов и развивал метод Фридрихса [1] для операторов с дискретным спектром. Следует отметить, что метод подобных операторов берет свое начало с работ Пуанкаре, Ляпунова, Крылова, Богомолова, Фридрихса, Тернера (теорема 1) и окончательно оформляется в работах Баскакова [2]-[4]. В статьях [2]-[4] развита техника, приспособленная к возмущенным неограниченным операторам, что вполне естественно в связи с приложениями к дифференциальным операторам. Однако рассматриваемые возмущения, хотя они могут быть и неограниченными операторами, подчинены невозмущенному оператору.

Отметим следуюший подход к определению сходящейся последовательности замкнутых операторов. Последовательность замкнутых операторов

$$
A_{n}: D\left(A_{n}\right) \subset H \rightarrow H, \quad n \geqslant 1
$$

назьвается сходящейся к оператору

$$
A_{0}: D\left(A_{0}\right) \subset H \rightarrow H
$$

если пересечение $K=\bigcap_{n \geqslant 1} \rho\left(A_{n}\right)$ их резольвентных множеств $\rho\left(A_{n}\right), n \geqslant 1$, непусто и для некоторого $\lambda_{0} \in K$ выполнено условие

$$
\lim _{n \rightarrow \infty}\left\|\left(A_{n}-\lambda_{0} I\right)^{-1}-\left(A_{0}-\lambda_{0} I\right)^{-1}\right\|=0 .
$$

Разумеется, здесь нет никаких дополнительных условий на $D\left(A_{n}\right), n \geqslant 1$. Этот подход к определению близости замкнутых операторов принуждает нас снова обратиться к теореме 1 и попытаться учесть разработанную в [2]-[5] технику метода подобных операторов.

Прежде чем изложить метод подобных операторов в удобной для данного конкретного случая форме, введем следующие обозначения. Через End $H$ обозначим банахово пространство линейных ограниченных операторов, действующих в $H$, и $\|\cdot\|_{\infty}-$ нормав End $H$. Термин “трансформатор”, следуя Крейну, далее будет употребляться для обозначения линейного оператора, действующего в пространстве линейных операторов. 
ОПРЕДЕЛЕниЕ 1. Два линейных оператора $A_{i}: D\left(A_{i}\right) \subset H \rightarrow H, i=1,2$, называются подобными, если существует непрерывно обратимьй оператор $U \in$ End $H$ такой, что

$$
U D\left(A_{2}\right)=D\left(A_{1}\right), \quad A_{1} U x=U A_{2} x \quad \forall x \in D\left(A_{2}\right) .
$$

Оператор $U$ называется оператором преобразования оператора $A_{1}$ в оператор $A_{2}$.

ОПРЕДЕЛЕНИЕ 2. Пусть $M$ - линейное многообразиеоператоров из End $H$ и $J: M \rightarrow$ $M, \Gamma: M \rightarrow$ End $H$ - трансформаторы. Тройка $(M, J, \Gamma)$ назьвается допустимой тройкой для оператора $A$, а $M$ - допустимым пространством возмущений, если

1) $M$ - банахово пространство со своей нормой, непрерьвно вложенное в End $H$, т.e.

$$
\|X\| \geqslant \text { const }\|X\|_{\infty} \quad \forall X \in \text { End } H
$$

2) $J$ и $\Gamma$ - непрерьвные операторы;

3) $A \Gamma X-\Gamma X A=X-J X$ для всех $X \in M$;

4) $X(\Gamma Y),(\Gamma X) Y \in M$ для всех $X, Y \in M$ и существует такая постоянная $\gamma>0$, что $\|$ Г $\|<\gamma$ и

$$
\max \{\|X(\Gamma Y)\|,\|(\Gamma Y) X\|\} \leqslant \gamma\|X\| \cdot\|Y\| \quad \forall X, Y \in M .
$$

Зафиксируем теперь некоторую допустимую для оператора $A$ тройку $(M, J, \Gamma)$ и возмутим оператор $A$ некоторым оператором $B$ из пространства допустимых возмущений $M$.

Теорема 2 [3]. Пусть выполнено условие $\|B\| \gamma<1 / 4$. Тогда операторы $A-B$ u $A-J X$ подобны, m.e.

$$
(A-B)(I+\Gamma X)=(I+\Gamma X)(A-J X)
$$

где $X \in M$ есть решение нелинейного операторного уравнения

$$
X=B \Gamma X-(\Gamma X) J X+B
$$

и его мохнно найти методом последовательных приближений, используя в качестве первого приближения нулевой оператор.

Теперь сделаем ряд предположений относительно невозмущенного оператора $A$ и возмущения $B$ и докажем теоремы о подобии возмущенного оператора $A-B$ оператору более простой структуры.

Пусть $A$ - нормальный компактный оператор, действующий в сепарабельном гильбертовом пространстве $H$. Пусть спектр $\sigma(A)$ оператора $A$ представим в виде

$$
\sigma(A)=\bigcup_{n} \sigma_{n}, \quad \sigma_{n} \cap \sigma_{m}=\varnothing, \quad n \neq m,
$$

где $\sigma_{n}, n \geqslant 1,-$ конечные подмножества.

Обозначим через $r_{n}$ расстояние от $\sigma_{n}$ до множества $\sigma(A) \backslash \sigma_{n}$, т.e. $r_{n}=\operatorname{dist}\left(\sigma_{n}\right.$, $\left.\sigma(A) \backslash \sigma_{n}\right)$. Символом $P\left(\sigma_{n}, A\right)$ или, короче, $P_{n}$ обозначим проектор Рисса, построенньй по спектральному множеству $\sigma_{n}$. Каждому оператору $X \in \operatorname{End} H$ поставим в 
соответствие матрищу $\widetilde{X}=\left(X_{i j}\right)$, составленную из операторных блоков $X_{i j}=P_{i} X P_{j}$. Сохраним обозначение $R^{-1}$ для ограниченного нормального оператора, определяемого следуюшим образом:

$$
R^{-1}=\sum_{i=1}^{\infty} r_{i} P_{i} .
$$

Возмутим оператор $A$ оператором $B \in$ End $H$ таким, что $R B \in \sigma_{2}(H)$, но запишем его в виде

$$
B=R^{-1} B_{0}
$$

$B_{0}=R B \in \sigma_{2}(H)$. Очевидно также, что $P_{i} B_{0} P_{j} \in \sigma_{2}(H)$ для всех $i, j \geqslant 1$.

Так как проекторы $\left\{P_{j}\right\}, j \geqslant 1$, являются ортопроекторами, то норма в $\sigma_{2}(H)$ определяется формулой

$$
\|X\|_{2}^{2}=\sum_{i, j \geqslant 1}\left\|P_{i} X P_{j}\right\|_{2}^{2} \quad \forall X \in \sigma_{2}(H)
$$

Зафиксируем также две ненулевые последовательности неотрицательных чисел $\alpha=$ $\left(\alpha_{n}\right), \beta=\left(\beta_{n}\right)$, принадлежащие пространству $l_{2}$. Символом $\sigma_{2}(H, \alpha, \beta)$ обозначим множество операторов из $\sigma_{2}(H)$ таких, что $\left\|X_{i j}\right\|_{2} \leqslant C \alpha_{i} \beta_{j}, i, j \geqslant 1$, с нормой

$$
\|X\|_{\alpha \beta}=\inf \left\{\text { const: }\left\|X_{i j}\right\| \leqslant \operatorname{const} \alpha_{i} \beta_{j} \quad \forall i, j\right\} \text {. }
$$

Основная схема построения используемых далее допустимых троек состоит в следующем. Во всех рассматриваемых тройках $(M, J, \Gamma)$ трансформатор $J: M \rightarrow M$ определяется формулой

$$
J X=\sum_{i=1}^{\infty} P_{i} X P_{i} \quad \forall X \in M_{2} .
$$

Конкретньй выбор пространства допустимых возмущений гарантирует сходимость этого ряда в сильной операторной топологии. Трансформатор Г определяется с помощью задания его операторных блоков. В качестве $\Gamma X_{m n}$ для $X_{m n}$ возьмем оператор $Y_{m n}$, удовлетворяющий условию $P_{m} Y_{m n} P_{n}=Y_{m n}$ и являющийся решением уравнения

$$
A Y_{m n}-Y_{m n} A=A_{m} Y_{m n}-Y_{m n} A_{n}=P_{m} X_{0} P_{n}
$$

где $A_{j}=P_{j} A, j \geqslant 1$ (см. [4]). При конкретном выборе пространства допустимых возмущений $M$ нам каждый раз придется доказьвать ограниченность трансформатора $\Gamma$, восстанавливая его из операторных блоков $\Gamma X_{m n}$.

Теорема 3. Пусть спектр оператора $A$ представим в виде (3) и возмущение $B \in$ End $H$ таково, что $B=R^{-1} B_{0}, B_{0} \in \sigma_{2}(H)$. Тогда при выполнении условия

$$
\left\|B_{0}\right\|_{2}<\frac{1}{4}
$$

оператор $A-B$ подобен оператору

$$
A-\sum_{i=1}^{\infty} P_{i} X P_{i}
$$


где $X$ - решение уравнения (2), причем $X=R^{-1} X_{0}, X_{0} \in \sigma_{2}(H)$. Oператор преобразования $U$ оператора $A-B$ в оператор $A-\sum_{i=1}^{\infty} P_{i} X P_{i}$ представлен $в$ виде $U=(I+\Gamma X)$, где ГX такжсе принадлежит идеалу $\sigma_{2}(H)$.

Более того, если $B_{0} \in \sigma_{2}(H, \alpha, \beta) u$

$$
\max \left\{\max _{j} \sum_{k \neq j} \alpha_{k} \beta_{k}, \sum_{m, n \geqslant 1} \alpha_{m}^{2} \beta_{n}^{2}\right\}\left\|B_{0}\right\|_{\alpha \beta}<\frac{1}{4}
$$

то $X_{0} \in \sigma_{2}(H, \alpha, \beta)$ и $X \in \sigma_{2}(H, \alpha, \beta)$, т.е. имеют место неравенства

$$
\begin{aligned}
\left\|X_{0 i j}\right\|_{2} \leqslant C_{2} \alpha_{i} \beta_{i}, & i, j \geqslant 1 \\
\left\|\Gamma X_{i j}\right\|_{2} \leqslant C_{3} \alpha_{i} \beta_{j}, & i, j \geqslant 1 .
\end{aligned}
$$

ДокАЗАТЕЛЬСТво. Построим сначала допустимую для оператора $A$ тройку. В качестве пространства допустимых возмущений $M$ возьмем пространство операторов, представимых в виде $X=R^{-1} X_{0}, X_{0} \in \sigma_{2}(H)$ с нормой $\|X\|_{M}=\left\|X_{0}\right\|_{2}$. Так как далее у нас появятся и другие пространства допустимых возмущений, то обозначим это пространство $M_{2}$.

Выполнение аксиомы 1 ) определения 2 следует из [1, с. 536], где показано, что $M_{2}-$ банахово пространство, и из очевидных неравенств

$$
\|X\|_{\infty}=\left\|R^{-1} X_{0}\right\|_{\infty} \leqslant\left\|R^{-1}\right\|_{\infty}\left\|X_{0}\right\|_{2}=\text { const } \cdot\|X\|_{M_{2}} .
$$

Перейдем к построению трансформаторов

$$
J: M_{2} \rightarrow M_{2}, \quad \Gamma: M_{2} \rightarrow \sigma_{2}(H) .
$$

Трансформатор $J$ зададим формулой (6). Из [4] следует, что уравнения (7), с помощью которых определяются операторные блоки оператора $\Gamma X$, разрешимы и $Y_{m n}$ допускает оценку

$$
\left\|Y_{m n}\right\|_{2} \leqslant\left(\operatorname{dist}\left(\sigma_{m}, \sigma_{n}\right)\right)^{-1}\left\|P_{m} X P_{n}\right\|_{2} \text {. }
$$

Теперь оператор $\Gamma X$ зададим формулой

$$
\Gamma X=\sum_{m, n} \Gamma\left(P_{m} X P_{n}\right)
$$

считая $\Gamma\left(P_{n} X P_{n}\right)=0$ для всех $n \geqslant 1$. Так как

$$
\begin{aligned}
\|\Gamma X\|_{2}^{2} & \leqslant \sum_{m, n}\left\|\Gamma X_{m n}\right\|_{2}^{2} \leqslant \sum_{m, n}\left(\operatorname{dist}\left(\sigma_{m}, \sigma_{n}\right)\right)^{-1} r_{m}\left\|X_{0 m n}\right\|_{2}^{2} \\
& \leqslant \sum_{m, n}\left\|X_{0 m n}\right\|_{2}^{2} \leqslant\left\|X_{0}\right\|_{2}^{2}=\|X\|_{M_{2}}^{2},
\end{aligned}
$$

то $\Gamma X \in \sigma_{2}(H)$ и $\|\Gamma X\| \leqslant\|X\|_{M_{2}}$.

Проверим вьполнение аксиомы 4) определения 2. Пусть

$$
Y \in M_{2}, \quad X \in M_{2}, \quad Z=(\Gamma Y) X=R^{-1} R \Gamma Y R^{-1} X_{0}=R^{-1} Z_{0},
$$


где $Z_{0}=R \Gamma Y R^{-1} X_{0}$. Покажем, что $Z \in M_{2}$, и оценим его норму:

$$
\begin{aligned}
\left\|Z_{0 i j}\right\|_{2} & \leqslant\left\|\sum_{k} P_{i} R \Gamma Y R^{-1} P_{k} X_{0} P_{j}\right\|_{2} \\
& \leqslant \sum_{k}\left|r_{i}^{-1}\left(\operatorname{dist}\left(\sigma_{i}, \sigma_{k}\right)\right)^{-1} \cdot r_{i}\right| \cdot\left\|Y_{0 i k}\right\|_{2} \cdot r_{k} \cdot\left\|X_{0 k j}\right\|_{2} \leqslant \sum_{k}\left\|Y_{0 i k}\right\|_{2} \cdot\left\|X_{0 k j}\right\|_{2} \\
\left\|Z_{0}\right\|_{2}^{2} & \leqslant \sum_{i, j}\left(\sum_{k}\left\|Y_{0 i k}\right\|_{2} \cdot\left\|X_{0 k j}\right\|_{2}\right)^{2} \leqslant \sum_{i, j}\left(\sum_{k}\left\|Y_{0 i k}\right\|_{2}^{2} \sum_{k}\left\|X_{0 k j}\right\|_{2}^{2}\right) \\
& \leqslant \sum_{i, k}\left\|Y_{0 i k}\right\|_{2}^{2} \cdot \sum_{k, j}\left\|X_{0 k j}\right\|_{2}^{2}=\|X\|_{M_{2}}^{2} \cdot\|Y\|_{M_{2}}^{2} .
\end{aligned}
$$

Таким образом, $Z \in M_{2}$ и

$$
\|(\Gamma Y) X\|_{M_{2}} \leqslant\|X\|_{M_{2}}^{2} \cdot\|Y\|_{M_{2}}^{2}
$$

Для оператора $X \Gamma Y$ доказательство очевидно, причем $\gamma=1$. Все аксиомы допустимой тройки имеют место. Условие (8) гарантирует разрешимость нелинейного операторного уравнения (2). Осталось применить теорему 2.

Для доказательства второго утверждения теоремы 3 сконструируем другую допустимую тройку следующим образом. Оператор $X \in$ End $H, X=R^{-1} X_{0}, X_{0} \in \sigma_{2}(H)$, отнесем к пространству допустимых возмущений $M_{\alpha \beta}$, если $X_{0} \in \sigma_{2}(H, \alpha, \beta)$, с нормой $\|X\|_{M_{\alpha \beta}}=\left\|X_{0}\right\|_{\alpha \beta}$. Относительно введенной нормы $M_{\alpha \beta}$ есть банахово пространство [4], и так как

$$
\begin{aligned}
\|X\|_{\infty} & =\left\|R^{-1} X_{0}\right\|_{\infty} \leqslant\left\|R^{-1}\right\|_{\infty} \cdot\left\|X_{0}\right\|_{2} \\
& =\left\|R^{-1}\right\| \cdot\|X\|_{M_{\alpha \beta}} \sqrt{\sum_{i, j} \alpha_{i}^{2} \beta_{i}^{2}}=\text { const }\|X\|_{M_{\alpha \beta}},
\end{aligned}
$$

то аксиома 1) вьполнена. Трансформаторы $J$ и Г строятся так же, как и в предыдущей допустимой тройке. Оценим норму оператора $\Gamma X$ :

$$
\begin{aligned}
\|\Gamma X\|_{2}^{2} & \leqslant \sum_{m, n}\left\|\Gamma P_{m} X P_{n}\right\|_{2}^{2} \leqslant \sum_{m, n}\left(\left(\operatorname{dist}\left(\sigma_{m}, \sigma_{n}\right)\right)^{-1} r_{m}\left\|X_{0 m n}\right\|_{2}^{2}\right) \\
& \leqslant\left(\sum_{m, n} \alpha_{m}^{2} \beta_{n}^{2}\right)^{2}\|X\|_{M_{\alpha \beta}}^{2}=\gamma_{1}^{2}\|X\|_{M_{\alpha \beta}}^{2} .
\end{aligned}
$$

Пусть теперь $X \in M_{\alpha \beta}, Y \in M_{\alpha \beta}$. Проверим выполнение аксиомы 4). Рассмотрим оператор $Z=X \Gamma Y=R^{-1} X_{0} \Gamma Y=R^{-1} Z_{0}$, покажем, что $Z \in M_{\alpha \beta}$ и получим оценку нормы этого оператора. Каждый оператор $Z_{0 m n}$ имеет вид

$$
Z_{0 m n}=\sum_{k} X_{0 m k} \Gamma Y_{k j}
$$


следовательно,

$$
\begin{aligned}
\left\|Z_{0 m n}\right\|_{2} & \leqslant \sum_{k \geqslant 1}\left\|X_{0 m k}\right\|_{2}\left\|\Gamma Y_{k n}\right\|_{2} \leqslant \sum_{\substack{k \geqslant 1 \\
k \neq j}}\left(\alpha_{m} \beta_{k} \alpha_{k} \beta_{n}\right)\|X\|_{M_{\alpha \beta}}\|Y\|_{M_{\alpha \beta}} \\
& =\left(\sum_{\substack{k \geqslant 1 \\
k \neq j}} \alpha_{k} \beta_{k}\right) \alpha_{m} \beta_{n}\|X\|_{M_{\alpha \beta}}\|Y\|_{M_{\alpha \beta}}=\gamma_{2} \alpha_{m} \beta_{n}\|X\|_{M_{\alpha \beta}}\|Y\|_{M_{\alpha \beta}} .
\end{aligned}
$$

Аналогично доказывается, что $(\Gamma Y) X \in M_{\alpha \beta}$ и

$$
\|(\Gamma Y) X\|_{M_{\alpha \beta}} \leqslant \gamma_{2}\|X\|_{M_{\alpha \beta}}\|Y\|_{M_{\alpha \beta}} .
$$

Таким образом, аксиома 4) имеет место и в качестве постоянной $\gamma$ можно взять величину

$$
\gamma=\max \left\{\max _{j} \sum_{k \neq j} \alpha_{k} \beta_{k}, \sum_{m, n} \alpha_{m}^{2} \beta_{n}^{2}\right\} .
$$

В этом случае утверждение теоремы 3 следует из теоремы 2.

\section{Теорема доказана.}

СлЕДСТВИЕ 2. Пусть все собственные значения $\lambda_{i}, i \geqslant 1$, оператора $A$ простые, соответствующие собственные векторы образуют ортонормированныи базис, $P_{i} x=\left(x, e_{i}\right) e_{i}$ для любого $x \in H$ и оператор $R^{-1} \in$ End $H$ задан формулой

$$
R^{-1} x=\sum_{n=1}^{\infty} r_{n} x_{n} e_{n}, \quad \text { если } \quad x=\sum_{n=1}^{\infty} x_{n} e_{n} .
$$

Пусть возмущение $B \in$ End $H$ таково, что $B=R^{-1} B_{0}, B_{0} \in \sigma_{2}(H)$. Тогда при выполнении условия

$$
4\left\|B_{0}\right\|<1
$$

оператор $A-B$ подобен оператору

$$
A-\sum_{i=1}^{\infty} P_{i} X P_{i}
$$

имеющему матрииу диагональной структуры в базисе $\left\{e_{i}\right\}, i \geqslant 1$, әде $X=R^{-1} X_{0}$, $X_{0} \in \sigma_{2}(H),-$ решение уравнения (2). Оператор преобразования $U$ таков, что $U=(I+\Gamma X)$, әде ГX также принадлежит идеалу $\sigma_{2}(H)$.

Более того, если выполнены условия

$$
\begin{gathered}
\left|b_{0 i j}\right| \leqslant c_{1} \alpha_{i} \beta_{j}, \quad i, j \geqslant 1, \quad c_{1} \geqslant 0, \\
4 \max \left\{\max _{j} \sum_{k \neq j} \alpha_{k} \beta_{k}, \sum_{m, n \geqslant 1} \alpha_{m}^{2} \beta_{n}^{2}\right\}\left\|B_{0}\right\|_{\alpha \beta}<1,
\end{gathered}
$$

то для әлементов матрицы операторов $X_{0}, \Gamma X$ имеют место оценки

$$
\begin{aligned}
& \left|X_{0 i j}\right| \leqslant c_{2} \alpha_{i} \beta_{i}, \quad i, j, \geqslant 1, \\
& \left|\Gamma X_{i j}\right| \leqslant c_{3} \alpha_{i} \beta_{j}, \quad i, j, \geqslant 1 .
\end{aligned}
$$


ДоКАЗАТЕЛЬСТво следствия 1 полностью повторяет доказательство теоремы 3 , кроме построения трансформатора $Г$. Остановимся только на этом вопросе.

Пусть $Y=\Gamma X$. Из равенства $A Y-Y A=X-J X$ следует, что элементы матрицы оператора $Y$ относительно базиса $e_{n}$ имеют вид

$$
Y_{i j}= \begin{cases}\frac{r_{i} X_{0 i j}}{\lambda_{i}-\lambda_{j}}, & i \neq j \\ 0, & i=j\end{cases}
$$

и

$$
\|\Gamma X\|_{2}^{2}=\sum_{i, j \geqslant 1}\left(\frac{r_{i} X_{0 i j}}{\lambda_{i}-\lambda_{j}}\right)^{2} \leqslant \sum_{i, j \geqslant 1} X_{0 i j}^{2}=\left\|X_{0}\right\|_{2}=\|X\|_{M} .
$$

Остальная часть доказательства не меняется. Следствие доказано.

СлЕДСТВИЕ 3. Пусть выполнены условия (10), (11) следствия 2. Тогда оператор $A-B$ является спектральным оператором скалярного типа (по Данфорду) $u$ для его собственных значений $\tilde{\lambda}_{i}$ и собственных векторов ё ${ }_{i}$ справедливы формуль

$$
\begin{gathered}
\tilde{e}_{i}=e_{i}+\Gamma X e_{i} \\
\tilde{\lambda}_{i}=\lambda_{i}-\left(B e_{i}, e_{i}\right)-\left(B \Gamma X e_{i}, e_{i}\right),
\end{gathered}
$$

причем

$$
\begin{aligned}
& \tilde{e}_{i}=e_{i}+\Gamma B e_{i}+O\left(\left\|B_{0}\right\|_{2}^{2}\right), \\
& \tilde{\lambda}_{i}=\lambda_{i}-\left(B e_{i}, e_{i}\right)-\left(B \Gamma B e_{i}, e_{i}\right)+O\left(\left\|B_{0}\right\|_{2}^{3}\right)
\end{aligned}
$$

$u$

$$
\sin \left(e_{i}, \tilde{e}_{i}\right)=\frac{\left\|\Gamma X e_{i}\right\|}{\sqrt{1+\left\|\Gamma X e_{i}\right\|^{2}}} \leqslant \frac{2.35\left\|\Gamma B e_{i}\right\|}{\sqrt{1+2.35^{2}\left\|\Gamma B e_{i}\right\|^{2}}} .
$$

ДокАЗАТЕЛЬСтво. Поскольку оператор $A-B$ подобен нормальному оператору $A-J X$, то $A-B$ - спектральный оператор скалярного типа. Непосредственной проверкой легко убедиться, что вектор $\tilde{e}_{i}$, определяемьй формулой (13), является собственным вектором оператора $A-B$, отвечающим собственному значению $\tilde{\lambda}_{i}$. Формулы (16), (15) получаются из (14) и (13), если в качестве неизвестного оператора $X$ взять второе приближение к нему по методу простых итераций. При выводе формулы (17) учтено, что отображение $\Phi(X)$, определенное правой частью уравнения (2), является сжимающим в шаре с центром в нуле и радиусом $r=2.35\|B\|$ (см. [2]). Следствие доказано.

СлЕДСТВИЕ 4. Пусть выполнены условия (10) и (11) следствия 2. Тогда векторы $е_{i}$ и $\tilde{e}_{i}$ связаны равенством:

$$
\tilde{e}_{i}=e_{i}+\sum_{i \neq k} \delta_{i} e_{i}
$$

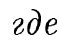

$$
\left|\delta_{i}\right| \leqslant \text { const }\left|\frac{r_{i}}{\lambda_{i}-\lambda_{k}}\right| \alpha_{i} \beta_{k}
$$


ДокАЗАТЕЛЬСТво. Формула (18) немедленно следует из (12) и (13).

Рассмотрим теперь несколько другой тип возмущения. Пусть $B=R^{-1} B_{0}$, где $B_{0}-$ ограниченньй линейньй оператор.

Введем в рассмотрение функцию $\alpha: \mathbb{Z} \rightarrow \mathbb{R}^{+}$, удовлетворяющую условиям

1) $\alpha(0)=1$;

2) $\sum_{n} \alpha(n)=M_{1}<\infty$;

3) $\sum_{k} \alpha(i-k) \alpha(k-j) \leqslant M_{2} \alpha(i-j), M_{2}>0$.

Определим подалгебру $\operatorname{End}_{\alpha} H$ операторов из End $H$ следующим образом. Оператор $X \in \operatorname{End} H$ отнесем к $\operatorname{End}_{\alpha} H$, если существует константа $c$ такая, что имеют место оценки

$$
\left\|X_{i j}\right\|_{\infty}=\left\|P_{i} X P_{j}\right\|_{\infty} \leqslant c \alpha(i-j)
$$

при этом норма определяется так:

$$
\|X\|_{*}=\inf \left\{c:\left\|X_{i j}\right\|_{\infty} \leqslant c \alpha(i-j) \quad \forall i, j \geqslant 1\right\}
$$

С учетом условий 1)-3) на функцию $\alpha$ легко показать, что $M_{*}$ является банаховой алгеброй.

Следуя терминологии из [6], можно сказать, что $M_{*}$ состоит из операторов $X \in$ End $H$, матричные элементы $X_{i j}$ которых убьвают по закону, определяемому функцией $\alpha$.

Теорема 4. Пусть спектр нормального компактного оператора $A$ представим в виде $(3), P_{i}=P\left(\sigma_{i}, A\right), B=R^{-1} B_{0}, B_{0} \in$ End $H$ и выполняется неравенство

$$
5\left\|B_{0}\right\|_{\infty}<1
$$

Тогда оператор $A-B$ подобен оператору $A-\sum_{i=1}^{\infty} P_{i} X P_{i}$, где оператор $X$, являющ,ийся решением уравнения (2), также имеет вид $X=R^{-1} X_{0} u X_{0} \in$ End $H$.

Более того, при выполнении условий

$$
\begin{gathered}
\left\|B_{0 i j}\right\|_{\infty} \leqslant c_{1} \alpha(i-j) \quad \forall i, j \geqslant 1, \quad c_{1}>0 \\
\max \left\{M_{1}, M_{2}\right\} \cdot\left\|B_{0}\right\|_{*}<\frac{1}{4}
\end{gathered}
$$

оператор $X=R^{-1} X_{0}$ maкой, что

$$
\left\|X_{0 i j}\right\|_{\infty} \leqslant c_{2} \alpha(i-j) \quad \forall i, j \geqslant 1, \quad c_{2}>0
$$

$u$

$$
\left\|\Gamma X_{i j}\right\|_{\infty} \leqslant c_{3} \alpha(i-j) \quad \forall i, j \geqslant 1, \quad c_{3}>0 .
$$


ДоказАтЕльство. Докажем сначала первое утверждение теоремы 4. Допустимым пространством возмущений $M_{\infty}$ в этом случае удобно взять пространство операторов вида $X=R^{-1} X_{0}, X_{0} \in$ End $H$ с нормой $\|X\|_{M_{\infty}}=\left\|X_{0}\right\|_{\infty}$. Тогда вьполнение аксиомы 1 ) определения 2 очевидно. Трансформатор $J: M_{\infty} \rightarrow M_{\infty}$ так же зададим формулой (6) и $\Gamma X$ так же определим на операторных блоках $\Gamma X_{m n}$, но в этом случае трансформатор $\Gamma$ действует из $M_{\infty}$ в End $H$. Тогда для оператора $\Gamma X_{m n}$ имеет место оценка

$$
\left\|\Gamma X_{m n}\right\|_{\infty} \leqslant 5 r_{m}\left(\operatorname{dist}\left(\sigma_{m}, \sigma_{n}\right)\right)^{-1}\left\|X_{0 m n}\right\|_{\infty} .
$$

Так как (см. [6, теорема 1.6]) любой оператор $Z \in$ End $H$ можно представить как

$$
Z=\lim _{n \rightarrow \infty} \sum_{|p| \leqslant n}\left(1-\frac{|p|}{n}\right) Z_{p}, \quad Z_{p}=\sum_{i-j=p} Z_{i j}
$$

и норма

$$
\left\|Z_{p}\right\|_{\infty}=\sup _{i-j=p}\left\|Z_{i j}\right\|_{\infty}
$$

то для оператора $\Gamma X$ имеем

$$
\begin{aligned}
\left\|\Gamma X_{p}\right\|_{\infty} & \leqslant 5 \sup _{m-n=p} r_{m}\left(\operatorname{dist}\left(\sigma_{m}, \sigma_{n}\right)\right)^{-1}\left\|X_{0 m n}\right\|_{\infty} \\
& \leqslant 5 \sup _{m-n=p}\left\|X_{0 m n}\right\|_{\infty}=5\left\|X_{0 p}\right\|_{\infty}, \\
\|\Gamma X\|_{\infty} & \leqslant 5 \sum_{p}\left\|X_{0 p}\right\|_{\infty}=5\left\|X_{0}\right\|_{\infty} .
\end{aligned}
$$

Следовательно, ряд $\sum_{p=-\infty}^{\infty} \Gamma X_{p}$ абсолютно сходится в End $H$ по операторной норме и оператор $\Gamma: M_{\infty} \rightarrow$ End $H$ построен. Проверим выполнение свойств допустимой тройки, относящихся к нему.

Пусть $X \in M_{\infty}, Y \in M_{\infty}$, тогда очевидно, что $X(\Gamma Y) \in M_{\infty}$ и

$$
\|X(\Gamma Y)\|_{M_{\infty}} \leqslant\|X\|_{M_{\infty}}\|Y\|_{M_{\infty}} .
$$

Рассмотрим теперь оператор $(\Gamma Y) X$, представив его в виде

$$
Z=(\Gamma Y) X=R^{-1} R \Gamma Y X=R^{-1} Z_{0} ;
$$

покажем, что $Z_{0} \in \operatorname{End} H$, и посчитаем его норму. Так как

$$
\begin{aligned}
Z_{0 i j} & =\sum_{k}(R \Gamma Y)_{i k} X_{k j}=\sum_{k} R_{i i} \Gamma Y_{i k} R_{k k}^{-1} X_{k j}, \\
\left\|Z_{0 i j}\right\|_{\infty} & \leqslant 5 \sum_{k} r_{i} r_{i}^{-1}\left\|Y_{0 i k}\right\|_{\infty}\left(\operatorname{dist}\left(\sigma_{i}, \sigma_{k}\right)\right)^{-1} r_{k}\left\|X_{0 k j}\right\|_{\infty} \\
& \leqslant 5 \sum_{k}\left\|Y_{0 i k}\right\|_{\infty}\left\|X_{0 j k}\right\|_{\infty},
\end{aligned}
$$

и $Y_{0} \in$ End $H, X_{0} \in$ End $H$, то

$$
\left\|Z_{0}\right\|_{\infty} \leqslant 5\left\|Y_{0}\right\|_{\infty}\left\|X_{0}\right\|_{\infty} .
$$


Таким образом, все аксиомы допустимой тройки вьполнены и $\gamma=5$. Условие (19) обеспечивает выполнение условий теоремы 2 . Следовательно, $A-B$ подобен $A-J X$ и $X \in M_{\infty}$, т.е. $X=R^{-1} X_{0}$, где $X_{0} \in$ End $H$.

Докажем теперь второе утверждение теоремы 4.

Строим пространство допустимых возмущений для случая, если $B=R^{-1} B_{0}$ и элементы $P_{i} B_{0} P_{j}$ допускают оценку (20). Оператор $Y \in$ End $H$ отнесем к пространству $M_{\alpha}$, если $Y=R^{-1} Y_{0}$ и $Y_{0} \in \operatorname{End}_{\alpha} H$, с нормой $\|Y\|_{M_{\alpha}}=\left\|Y_{0}\right\|_{*}$. Проверку аксиомы 1) можно найти в [7] для пространства $\operatorname{End}_{\alpha} H$, а отсюда автоматически следует и выполнение ее и для пространства $M_{\alpha}$. Трансформаторы $J: M_{\alpha} \rightarrow M_{\alpha}$ и Г: $M_{\alpha} \rightarrow$ End $H$ строятся так, как показано вьше; при этом

$$
\begin{gathered}
\left\|\Gamma X_{p}\right\|_{\infty} \leqslant 5 \sup _{i-j=p}\left\|X_{0 i j}\right\|_{\infty} \leqslant 5\|X\|_{M_{\alpha}} \alpha(p), \\
\|\Gamma X\|_{\infty} \leqslant 5 \sum_{p}\|X\|_{M_{\alpha}} \alpha(p) \leqslant 5 M_{1}\|X\|_{M_{\alpha}}
\end{gathered}
$$

т.e. $\Gamma X \in \operatorname{End}_{\alpha} \mathrm{H}$.

Пусть теперь $X \in M_{\alpha}$ и $Y \in M_{\alpha}$. Тогда рассмотрим оператор

$$
Z=X \Gamma Y=R^{-1} X_{0} \Gamma Y=R^{-1} Z_{0}
$$

где

$$
\begin{aligned}
\left\|Z_{i j}\right\|_{\infty} & \leqslant \sum_{k}\left\|X_{0 i k}\right\|_{\infty}\left\|\Gamma Y_{k j}\right\|_{\infty} \\
& \leqslant 5 \sum_{k}\|X\|_{M_{\alpha}} \alpha(i-k)\|Y\|_{M_{\alpha}} \alpha(k-j) \leqslant 5 M_{2}\|X\|_{M_{\alpha}}\|Y\|_{M_{\alpha}}
\end{aligned}
$$

т.е. $Z_{0} \in \operatorname{End}_{\alpha} H$ и $\|X \Gamma Y\| \leqslant 5 M_{2}\|X\|_{M_{\alpha}}\|Y\|_{M_{\alpha}}$. Рассмотрим теперь оператор

$$
C=(\Gamma Y) X=R^{-1}(R(\Gamma Y)) R^{-1} X_{0}=R^{-1} C_{0}
$$

и оценим норму оператора $C_{0}$ :

$$
\begin{aligned}
\left\|C_{0 i j}\right\|_{\infty} & \leqslant \sum_{k}\left\|R_{i i} \Gamma \mathrm{X}_{i k} R_{k}^{-1} X_{0 k j}\right\| \\
& \leqslant 5 \sum_{k}\|X\|_{M_{\alpha}} \alpha(k-j)\|Y\|_{M_{\alpha}} \alpha(i-k) \leqslant 5 M_{2}\|X\|_{M_{\alpha}}\|Y\|_{M_{\alpha}} .
\end{aligned}
$$

Таким образом, все аксиомы допустимой тройки вьполнены и в этом случае, причем $\gamma=5 \max \left\{M_{1}, M_{2}\right\}$. Применяя теорему 2 , получаем, что операторы $A-B$ и $A-J X$ подобны, и так как $X \in M_{\alpha}$, то $X_{0} \in \operatorname{End}_{\alpha} H$.

Теорема 4 доказана.

ЗАмЕчАниЕ. Для данного типа возмущений также можно сформулировать и доказать результаты, аналогичные результатам следствий 2 и 3 теоремы 3 . Мы не приводим соответствующие формулировки ввиду их полной аналогии. 
СлЕДСТВИЕ 5. Пусть все собственные значения $\lambda_{i}, i \geqslant 1$, оператора $A$ простые и выполняются условия теоремы 4. Тогда собственные векторы $e_{k}, k \geqslant 1$, невозмущенного оператора $A$ и собственные векторы $\tilde{e}_{k}, k \geqslant 1$, возмущенного оператора $A-B$ связаны равенством

$$
\tilde{e}_{k}=e_{k}+\sum_{i \neq k} d_{i} e_{i}
$$

$2 \partial e$

$$
\left|d_{i}\right|=\mathrm{const}\left|\frac{r_{i}}{\lambda_{i}-\lambda_{k}}\right| \alpha(i-k) .
$$

Рассмотрим теперь обратную задачу спектрального анализа. Пусть известны некоторые оценки коэффициентов Фурье собственных векторов возмущенного оператора $A-B$ в базисе из собственных векторов невозмущенного оператора $A$, а сам оператор $B$ не известен. Приводимая ниже теорема 5 позволяет в этом случае определить некоторые характеристики оператора $B$.

Сначала наложим на функцию $\alpha: \mathbb{Z} \rightarrow \mathbb{R}^{+}$еще одно условие:

$$
\lim _{|k| \rightarrow \infty}|k|^{-1}|\ln \alpha(k)|=0
$$

и будем считать в дальнейшем его вьполненным. Это условие гарантирует наполненность банаховой алгебры $\operatorname{End}_{\alpha} H$ [6, теорема 1], т.е. каждый обратимьй в End $H$ опеpaтор $X \in \operatorname{End}_{\alpha} H$ обратим и в $\operatorname{End}_{\alpha} H$.

Tеорема 5. Пусть собственные значения $\lambda_{i}, i \geqslant 1$, оператора $A$ простые $u$ возмущение $B$ представимо в виде $B=R^{-1} B_{0}, B_{0} \in$ End $H$,

$$
5\left\|B_{0}\right\|_{\infty}<\frac{1}{4}
$$

Пусть собственные векторы $\tilde{e}_{k}, k \geqslant 1$, возмущенного оператора $A-B$ и собственные векторы $e_{k}, k \geqslant 1$, невозмущенного оператора $A$ связаны равенством

$$
\tilde{e}_{k}=e_{k}+\sum_{i \neq k} \beta_{i k} e_{i}
$$

причем

$$
\left|\beta_{i k}\right| \leqslant c \alpha(i-k) \gamma(i, k), \quad \gamma(i, k)=\frac{r_{i}}{\lambda_{i}-\lambda_{k}}
$$

Тогда для әлементов $b_{0 i j}$ матрицы оператора $B_{0}$ имеют место оценки

$$
\left|b_{0 i j}\right| \leqslant c \alpha(i-j), \quad c>0 .
$$


ДокАЗАТЕЛЬСтво. В качестве допустимой тройки в данном случае возьмем тройку $\left(M_{\infty}, J, \Gamma\right)$, построенную в теореме 4 . Тогда собственные векторы $\tilde{e}_{k}$ и $e_{k}$ связаны равенством

$$
\tilde{e}_{k}=e_{k}+\Gamma X e_{k}=e_{k}+\sum_{i \neq k}(\Gamma X)_{i k} e_{i} .
$$

Так как собственные векторы оператора $A$ (или оператора $A-J X$ ) образуют базис, а разложение любого вектора по базису единственно, то из (21) и (22) следует, что

$$
\left|\Gamma X_{i k}\right| \leqslant c \gamma(i, k) \alpha(i-k) .
$$

С другой стороны,

$$
(\Gamma X)_{i k}= \begin{cases}r_{i} \frac{X_{i k}}{\lambda_{i}-\lambda_{k}}, & i \neq k \\ 0, & i=k\end{cases}
$$

следовательно,

$$
X_{0 i j}=\frac{\lambda_{i}-\lambda_{k}}{r_{i}}(\Gamma X)_{i k}
$$

откуда

$$
\left|X_{0 i j}\right| \leqslant c \frac{\lambda_{i}-\lambda_{k}}{r_{i}} \cdot \frac{r_{i}}{\lambda_{i}-\lambda_{k}} \alpha(i-k) .
$$

Таким образом, $X_{0}$ есть элемент пространства $\operatorname{End}_{\alpha} H$ и

$$
X=R^{-1} X_{0} \in M_{\alpha} .
$$

Вернемся к нелинейному уравнению (2) и перепишем его в виде:

$$
B=(X+\Gamma X-J X)(I+\Gamma X)^{-1} \text {. }
$$

Так как $\Gamma X \in \operatorname{End}_{\alpha} H$ и $\operatorname{End}_{\alpha} H-$ наполненная алгебра, то $(I+\Gamma X)^{-1} \in \operatorname{End}_{\alpha} H$. Операторы, стоящие в правой части равенства (23), принадлежат $M_{\alpha}$, следовательно, $B \in M_{\alpha}$, т.е. $B=R^{-1} B_{0}, B_{0} \in \operatorname{End}_{\alpha} H$ и $\left|b_{0 i j}\right|<c \alpha(i-j)$.

Теорема доказана.

\section{СПИСОК ЦИТИРОВАННОЙ ЛИТЕРАТУРЫ}

[1] Данфорд Н., Шварц Дж. Т. Линейные операторы. Спектральные операторы. М.: Мир, 1974.

[2] Баскаков А. Г. Гармонический анализ линейных операторов. Учебное пособие. Воронеж: Изд-во Воронежского ун-та, 1987.

[3] Баскаков А. Г. Спектральный анализ возмущенных неквазианалитических и спектральных операторов // Изв. РАН. Сер. матем. 1994. Т. 58. № 4. С. 3-32.

[4] Баскаков А. Г. Спектральный анализ относительно конечномерных возмущений спектральных операторов // Изв. вузов. Матем. 1991. № 1. С. 3-11.

[5] Ускова Н. Б. О спектре некоторых классов дифференциальных операторов // Дифференц. уравнения. 1994. Т. 30. № 2. С. 350-352.

[6] Баскаков А. Г. Оценки элементов обратных матриц и спектральный анализ линейных операторов // Изв. РАН. Сер. матем. 1997. Т. 61. №6. С. 3-26.

[7] Ускова Н. Б. Об оценках спектральных проекторов возмущенных самосопряженных операторов // Сиб. матем. ж. 2000. Т. 41. № 3. С. 712-721. 